\title{
Analysis and research on wireless sensor networks
}

\author{
Huanan Zhang* \\ School of Data and Computer Science, Guangdong Peizheng College, Guangzhou, 510830, China
}

\begin{abstract}
With the development of sensor, computer and communication, the researcher attaches more and more attentions to the research of the wireless sensor networks because of its bright future in applications. This paper introduces the structure, characteristics of wireless sensor networks. Then, some key questions of the research field are discussed based on the basic principles. The end of paper discusses the application fields of wireless sensor networks and comes to a conclusion.
\end{abstract}

\section{Introduction}

With the development of Sensor, computer and Wireless communication, a new computer network, Wireless Sensor Networks(WSNs), appears. Because of its wide application prospect, wireless sensor networks have attracted more and more researchers' attention. Wireless sensor network is a wired or wireless network composed of a group of sensors in the form of Ad Hoc. Its purpose is to cooperatively perceive, collect and process the information of perceived objects in the geographical area covered by the sensor network and transmit it to observer. This kind of sensor network sensor technology, embedded computing technology and wireless communication technology, collaboration awareness, monitor and collect various perceived object under the environment of information, based on the information of the collaborative information processing, securing of perception object information accurately, and then transmitted Ad hoc way to need this information to the user. Since the hardware resources of wireless sensor networks are very limited and the working environment is usually limited by some resources, a lot of challenging research topics are proposed for theoretical researchers and engineering technicians. This paper introduces the structure and characteristics of wireless sensor as well as the volume structure of sensor network nodes, analyzes some key problems of wireless sensor network research from the basic principles of wireless sensor network design, and finally discusses the application prospect and development direction of wireless sensor network [1].

\section{Wireless sensor structure}

The basic elements of wireless sensor networks are nodes. At the same time, the node has the functions of transmission, information processing and wireless communication. The

\footnotetext{
*Corresponding author: topwn2008@163.com
} 
structure of nodes may be different for different application environments, but their basic composition is the same. A node usually consists of a sensor, microprocessor, storage, A/D conversion interface, wireless emission, and receiver and power supply. The node structure diagram of wireless sensor network is shown in Fig.1.

Wireless sensor nodes are usually distributed in a specific geographical area by air drop or preset, and then automatically configured and organized into a wireless network network. Nodes transmit the collected data to base stations/hostesses through multi-hop communication. Base stations are directly connected to the internet or communications satellites so that users can access the data remotely [2].

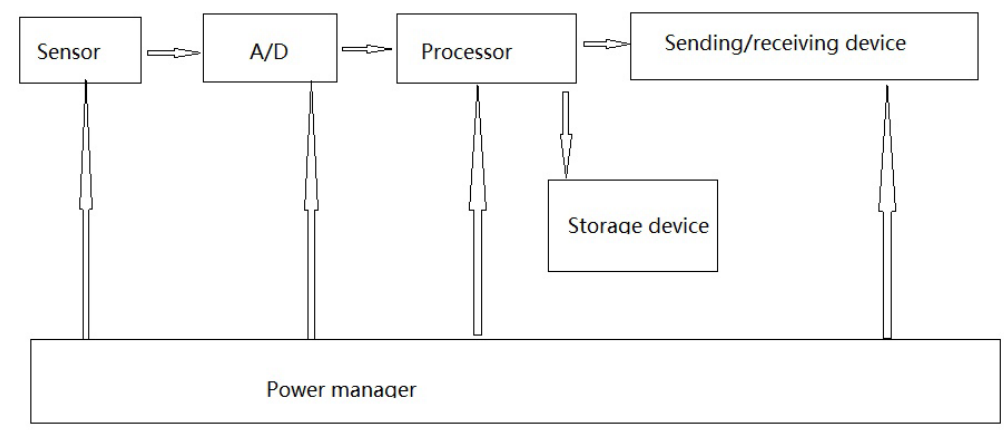

Fig. 1. Structure diagram of wireless sensor network nodes.

\section{Characteristics of wireless sensor network}

Traditional wireless network includes mobile communication network, wireless LAN, wireless personal network, Bluetooth network, etc. Their design is basically to meet people's various needs at the same time, such as voice, video, image and so on. However, wireless sensor networks are generally designed for a specific need. They are applicationbased wireless networks. As you can see from the above definition of the wireless sensor network, the wireless sensor network is a special type of Ad Hoc network. Therefore, in addition to the general characteristics of Ad Hoc networks, such as dynamic topology, nocenter self-organization, multi-hop routing, and limited energy and bandwidth, the wireless sensor network also has its own characteristics compared with Ad Hoc network:

(1) Resources are more limited. Due to the impact of price and hardware volume, the signal processing capacity and computing capacity of wireless sensor network nodes are worse, and the storage capacity is smaller.

(2) The network scale is larger and covers a wider geographical area. The order of magnitude of wireless sensor network nodes is usually hundreds or thousands, some even tens of thousands. In order to monitor a geographical area, hundreds of nodes are usually placed in that area. The nodes are more densely distributed.

(3) Sensor network nodes do not have a global identity, such as IP address. Each node only knows the location and identification of its neighboring nodes, and the sensor network carries out signal processing and communication through the cooperation between neighboring nodes, which has strong cooperation [3].

(4) The topological structure is relatively stable. Topology changes in Ad Hoc networks are primarily caused by the movement of nodes. However, the topology change of wireless sensor network is mainly caused by the exhaustion of node energy, and the node location is relatively fixed. 
(5) As the sensor node distribution is very dense, the perception data between adjacent nodes has a strong similarity.

(6) More limited energy. Due to the influence of hardware conditions, wireless sensor nodes are usually powered by batteries, and the power supply is more limited. Under the influence of energy, the communication distance between the nodes of the wireless sensor network is shorter, generally only a few tens of meters or even shorter.

(7) For some specific wireless sensor networks, the working environment is more severe. In addition, the distribution of sensor nodes is more dense, and the environmental noise interference and relative interference between nodes are stronger.

(8) Wireless sensor networks are based on data-centric routing, which is also different from the end-to-end communication of Ad Hoc.

It can be seen from the above that, although the wireless sensor network can be regarded as a special Ad hoc network, the characteristics of the wireless sensor network itself determine that the research results of Ad Hoc can only be used as a reference. People must research and develop new technologies suitable for wireless sensor networks [4].

\section{Some key problems of wireless sensor networks}

First, let's look at the protocol stack for wireless sensor networks. It consists of two planes: the communication plane and the management plane. The communication plane includes the physical layer, the data link layer, the network layer, the transport layer and the application layer. Management includes power management, mobile management and collaborative management. Communication plane realizes the information transfer between network nodes. The node passes the data it receives to the management plane, which determines how the node processes the data. The management plane is responsible for detecting and controlling nodes so that they work correctly [5].

\subsection{Physical layer}

The physical layer is mainly responsible for the collection of perception data and sampling of the collected data, signal modulation and demodulation, signal transmission and reception, power control and other tasks. The energy required to transmit and receive the same length of bit data. Considering that the energy of wireless sensor network nodes is very limited, energy saving is very important to extend the lifetime of the network. Therefore, high frequency can be used to transmit signals and low frequency to receive signals. How to manage and control dynamic power is a very important topic in wireless sensor networks.

\subsection{Data link layer}

The data link layer is responsible for the control of media access and the establishment of reliable communication links between nodes. It is mainly composed of the media access control MAC. The traditional MAC protocol based on competition mechanism is difficult to meet the needs of wireless sensor networks. Because content-based MAC protocols require multiple handshakes, there is a high risk of data collisions, creating a waste of energy that is not desirable in wireless sensor networks. Therefore, MAC protocols for wireless sensor networks generally adopt pre-planned mechanisms such as TDMA to protect node energy. MAC layer is one of the research hotspots in wireless sensor networks. 


\subsection{Routing layer}

The primary task of the network layer is to discover and maintain routing. Because multihop communication is more energy efficient than direct communication, it also meets the needs of data fusion and collaborative signal processing.

In wireless sensor networks, nodes generally adopt multi-hop routing to connect source and host. However, the existing Ad Hoc network multi-hop routing protocols, such as AODV, TORA and DSR, are generally not suitable for the characteristics of wireless sensor networks. Wireless sensor networks must develop their own routing protocols. In fact, because wireless sensor networks have a strong specific application background, a sensor network is usually designed for a specific application. Therefore, it is difficult to adopt a common routing protocol. Different from the traditional address-centered routing protocol, the routing protocol of wireless sensor networks is data-centered and does not have a global identity. It is generally a attribute-based addressing mode and usually adopts the on-demand passive routing mode.

\subsection{Collaborative signal processing}

In wireless sensor networks, the energy of nodes is very limited, and nodes must complete multiple tasks such as sensing data, signal processing and communication with neighboring nodes. In this way, how to effectively save energy is crucial. Effective perceptual model, low sampling rate and low power consumption signal processing algorithm must be adopted. At the same time, in order to effectively complete the monitoring of the perception area, including target detection, classification, recognition and tracking, signal processing must be completed within a certain period of time. In addition, in wireless sensor network, the communication link of nodes is very unstable. Because of the advantages of low delay, strong robustness and measurability of collaborative signal processing, it is necessary to carry out collaborative signal processing between wireless sensor network nodes. Collaborative signal processing refers to the collaborative processing of data from multiple sources by multiple nodes.

From another point of view, the traditional centralized signal processing is not suitable for the needs of wireless sensor networks. In previous wireless communication systems, network nodes send the collected raw data directly to the central node, and the central node performs signal processing tasks. In the case of wireless sensor networks where bandwidth is very valuable, this centralized processing method wastes a lot of bandwidth resources. Moreover, the nodes of the processing center attachments are likely to run out of energy because they have to forward a large amount of information, which will greatly shorten the network's life time.

In the centralized signal processing mode, the nodes directly transmit the raw data collected to the remote processing center, which is also not desirable in the wireless sensor network. Since the perception data of adjacent nodes in wireless sensor network usually has strong correlation, there is a lot of redundancy. If the original data is transmitted directly, limited bandwidth resources will be wasted. Cooperative signal processing technology should be used to fuse and compress the data between adjacent nodes before data transmission. In this way, the redundancy of data can be reduced and the overall traffic of the network can be reduced.

Collaborative signal processing is an on demand, target-oriented signal processing method. Only when nodes receive specific query tasks, they will carry out the signal processing tasks related to the current query. Collaborative signal processing is another multi-resolution signal processing method, which can process different signals according to different query tasks. The improvement of collaborative signal processing energy is 
actually based on the increase of information exchange between adjacent nodes. Therefore, a compromise between algorithm performance and network resources is often considered in the design of practical algorithms. Cooperative signal processing also faces some problems, such as the influence of doppler frequency shift caused by node movement and the time synchronization of nodes. Collaborative signal processing is a new technology, and there are still many problems to be discussed.

\subsection{Cross-layer design}

Many current wireless sensor network protocols are based on the traditional hierarchical structure, which is actually a local sub-optimal scheme. Since the resources of wireless sensor networks, such as energy, bandwidth and node resources, are very limited, such a hierarchical suboptimal structure is difficult to adapt to the development of wireless sensor networks.

It is a global optimization problem that cross-layer design satisfies the global needs through the information exchange between layers. Of course, cross-layer design does not mean that there is no longer a need for protocol specifications, nor does it mean that all specifications are integrated together. Cross-layer design is to optimize the performance of the whole network by sharing information between layers. Different network systems need different design schemes, and different applications also need different design schemes. Wireless sensor network is an application-based network. In particular, some applications do not need to involve all the network layer, such as multi-hop LPS(Local Positioning System) System training. In this way, when designing such a network system, irrelevant layers can be removed and only the optimization of other layers can be considered. The traditional hierarchical structure obviously cannot meet this requirement. On the other hand, the specific application environment of wireless sensor network often requires the network life time to be long enough and the network delay to be as short as possible, which is a contradiction with the limited energy supply, limited node resources and bandwidth of wireless sensor network, and the traditional hierarchical structure is difficult to solve this problem. Therefore, cross-layer design needs to be introduced. Especially in wireless communication environment such as wireless sensor network, because each layer is closely related, it is more necessary to consider the problem of cross-layer.

\subsection{Operating system}

Operating system support is one of the key technology of wireless sensor network (WSN), the traditional operating system, Windows and Unix obviously can't satisfy the need of wireless sensor network (WSN). People need to develop a new operating system, it need to be able to meet the needs of special wireless sensor networks, And need to be able to effectively make use of the wireless sensor network node limited hardware resources to provide service for applications. Department of computer science at the university of California, Berkeley meter "smart dust" group Based on multi-thread technology, developed a micro type of operating system, Tiny0S, is produced in order to adapt to this need. It is a framework Based on components (Component -based), able to quickly achieve a variety of applications. Another tiny wireless sensor network operating system is developed by the us at the University of Colorado MANTIS OS, It supports fast and flexible architecture of wireless sensor network (WSN). The operating system how to automatic configuration, adaptive to support the needs of the wireless sensor network, it is still an open research problem [6]. 


\section{Applications of wireless sensor network (WSN)}

Wireless sensor networks (WSNS) have attracted extensive attention from military, industrial and academic circles all over the world because of their potential military, industrial and civilian applications. Some of its important applications are:

\subsection{Military}

Military application is one of the important application of wireless sensor network. Under the condition of no infrastructure support, wireless sensor network can be configured to rapidly on self-organization network. As a result, wireless sensor network can decorate on the enemy's position, is used to collect information about the enemy of some important goals, and tracking the enemy's army.

\subsection{Environmental detection}

Environment detection is one of the early application of wireless sensor. Wireless sensor networks can be used to detect air quality and track pollution sources. Wireless sensor networks can also be used to provide early warning for chemical and biological disasters. In some emergency situations, such as fire, wireless sensors can also be used to determine the center and intensity of the fire.

\subsection{Civil}

Wireless sensor networks can also be used to build systems such as personal health and smart homes. Wireless sensor networks can also be widely used in transportation, architecture and space exploration.

\section{Conclusion}

Wireless sensor network (WSN) is an emerging network technology, and people still have a long way to go in its research. In this paper, some key technologies of wireless sensor networks are analyzed according to the special characteristics of wireless sensor networks. In view of the limited resources of wireless sensor networks, a compromise scheme should be found between algorithm performance and network resources when designing relevant algorithms for wireless sensor networks. In addition, as wireless sensor networks have a strong application environment, it is difficult to design common network protocols. Corresponding node structures and network architectures should be designed according to specific applications, and then corresponding network protocols should be designed according to specific situations.

\section{References}

1. Y. Wu, L. X. Yang, Y. Liu and W. Lou, "Energy-Efficient Wake-Up Scheduling for Data Collection and Aggregation," IEEE Transactions on Parallel and Distributed Systems, Vol. 21, No. 2, 2010, pp. 275-287.

2. Wang, Q.; Dai, H.-N.; Cheang, C.F.; Wang, H. Link Connectivity and Coverage of Underwater Cognitive Acoustic Networks under Spectrum Constraint. Sensors 2017, $17,2839$. 
3. Wang, Z.; Han, G.; Qin, H; Zhang, S.; Sui, Y. An energy-aware and void-avoidable routing protocol for underwater sensor networks. IEEE Access 2018, 6, 7792-7801.

4. Kumar, S.; Chaurasiya, V.K. A Strategy for Elimination of Data Redundancy in Internet of Things (IoT) Based Wireless Sensor Network (WSN). IEEE Syst. J. 2019, 13, 1650-1657.

5. Han, D., Du X and Y. Lu, Trustworthiness and a Zero Leakage OTMP-P2L Scheme Based on NP Problems for Edge Security Access. Sensors (Basel), 2020. 20(8).

6. Shang, X., et al., Secrecy Performance Analysis of Wireless Powered Sensor Networks Under Saturation Nonlinear Energy Harvesting and Activation Threshold. Sensors (Basel), 2020. 20(6). 